TITLE:

\title{
Fast ion charge exchange spectroscopy measurement using a radially injected neutral beam on the large helical device
}

\section{AUTHOR(S):}

Osakabe, Masaki; Murakami, Sadayoshi; Yoshinuma, Mikirou; Ida, Katsumi; Whiteford, Allan; Goto, Motoshi; Kato, Daiji; ... Tokuzawa, Tokihiko; Takeiri, Yasuhiko; Kaneko, Osamu

\section{CITATION:}

Osakabe, Masaki ...[et al]. Fast ion charge exchange spectroscopy measurement using a radially injected neutral beam on the large helical device. REVIEW OF SCIENTIFIC INSTRUMENTS 2008, 79(10): 10E519.

\section{ISSUE DATE:}

2008-10

\section{URL:}

http://hdl.handle.net/2433/84607

\section{RIGHT:}

Copyright 2008 American Institute of Physics. This article may be downloaded for personal use only. Any other use requires prior permission of the author and the American Institute of Physics. 


\title{
Fast ion charge exchange spectroscopy measurement using a radially injected neutral beam on the large helical device ${ }^{a)}$
}

\author{
Masaki Osakabe, Sadayoshi Murakami, ${ }^{\text {b) }}$ Mikirou Yoshinuma, Katsumi Ida, \\ Allan Whiteford, ${ }^{c}$ Motoshi Goto, Daiji Kato, Takako Kato, Kenichi Nagaoka, \\ Tokihiko Tokuzawa, Yasuhiko Takeiri, and Osamu Kaneko \\ National Institute for Fusion Science, Toki 509-5292, Japan
}

(Presented 12 May 2008; received 13 May 2008; accepted 14 July 2008; published online 31 October 2008)

\begin{abstract}
An experimental technique to investigate fast ion confinement based on charge exchange spectroscopy of $H_{\alpha}$-light was applied to evaluate the confinement property of perpendicular fast ions in large helical device (LHD). Sensitivities of the $H_{\alpha}$ spectra to the pitch angles of injected neutral beams (NBs) and these to the angle between the sight line of the measurement and NB injection path are examined. The energy dependence of the charge exchange cross section significantly affects the observed spectra since the driving NB is injected perpendicular to the magnetic field lines in the geometry of LHD. The measured spectra are compared to the spectra of GNET simulation results and the simulated spectra agreed well with the experimental measurement when we take into account the contribution of halo neutrals. Although it is difficult to obtain the fast ion distribution functions directly, this technique provides useful experimental data in benchmarking simulation codes.

(C) 2008 American Institute of Physics. [DOI: 10.1063/1.2968695]
\end{abstract}

\section{INTRODUCTION}

Confinement of fast ions is one of the most important issues in helical devices since a large helical ripple has a large influence on the topology of fast ion orbits. For the fast ions which have their large energy fraction perpendicular to magnetic field lines, the effect of the ripple becomes significant to their confinement properties. Considering the alphaparticle confinement in a fusion reactor relevant machine based on a helical configuration, such as force-free helical reactor (FFHR), ${ }^{1}$ the investigation of the confinement properties is important.

Recently, a radial neutral beam (NB) injector was installed on the large helical device (LHD). The NB injects hydrogen with an energy of $40 \mathrm{keV}$ and a power of $7 \mathrm{MW}$. The main aim of the NB is to use it as a heating tool of ions in LHD plasmas and as a diagnostic for charge exchange spectroscopy (CXS) measurement of ion temperatures. In addition, this NB can be also used as a probing beam to evaluate the alpha-particle confinement in FFHR since the normalized Larmour radii of the injected ions in LHD $\left(r_{L} / a=0.024\right.$ at $\left.B_{t}=2 \mathrm{~T}\right)$ are close to those of alpha particles in FFHR $\left(r_{L} / a=0.025\right)$.

On LHD, several neutral particle analyzers are installed to monitor the fast ion spectra at different regions in both real and phase space, ${ }^{2}$ but these are mostly equipped with no active neutral source. Therefore, it is very difficult to get fast

\footnotetext{
${ }^{\text {a) }}$ Contributed paper published as part of the proceedings of the 17th Topical Conference on High-Temperature Plasma Diagnostics, Albuquerque, New Mexico, May 2008.

b) Also at Department of Nuclear Engineering, Kyoto University, Kyoto 6068501, Japan.

${ }^{c}$ Also at Department of Physics, University of Strathclyde, 107 Rottenrow, Glasgow, G4 0NG, United Kingdom.
}

ion information in the core region from these measurements. ${ }^{3}$ However, it is desirable to have a diagnostic which indicates the behavior of fast ions in LHD.

In the present article, we will show our approach to evaluate the perpendicular fast ions using the CXS measurement of $H_{\alpha}$ light from hydrogen neutrals on LHD. The method is similar to the fast ion deuterium-alpha (FIDA) measurement, which was proposed and demonstrated by Heidbrink et al. ${ }^{4}$ The main difference is the geometry of NB injection and the measured ion species. The difference in injection geometry gives a different response of the Doppler shifted $H_{\alpha}$ spectra, which makes the interpretation of the measurement more difficult than their case. ${ }^{4}$ These difficulties are described in Sec. II, together with the experimental apparatus of the measurement on LHD. The initial experimental results being obtained for the LHD standard configuration are shown in Sec. III and are compared to the GNET simulation results.

\section{EXPERIMENTAL APPARATUS}

Figure 1 shows the experimental geometry of the fast ion CXS (FICXS) measurement on LHD.

As mentioned above, the basic premise of the measurement is very similar to the FIDA measurement at DIIID; the Doppler shifted components of $H_{\alpha}$ light from fast neutrals, which are produced by the charge exchange reaction between gyrating fast ions and injected NB ions, are observed. The major difference between our measurement and the FIDA (Ref. 4) measurement is the injection direction of the NB. Instead of using the tangential-NB as in DIIID, we are using a radial NB because the energy of our tangential-NB $(180 \mathrm{keV})$ is too high to use as an active neutral source for 


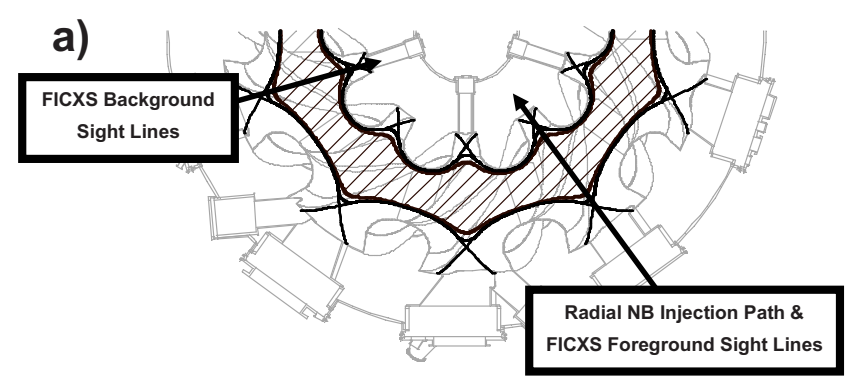

b)

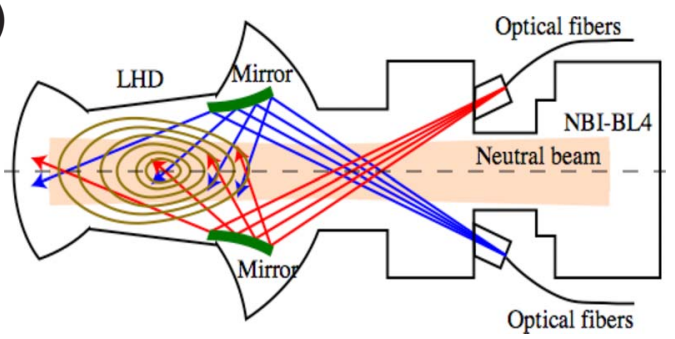

FIG. 1. (Color online) Schematic drawings of the LOSs for FICXS measurement and injection path of the radial-NB on LHD. Projected views onto (a) the midplane of LHD and onto (b) a poloidal cross section.

CXS measurements. The radial-NB, in contrast, can be operated with the energies of up to $40 \mathrm{keV}$ with hydrogen and up to $7 \mathrm{MW}$ of NB-injection power is possible from four ion sources. In the FICXS measurement, this NB is implicitly used as a source of perpendicular fast ions as well as the active neutral source of the measurement.

The optics normally used for bulk ion temperature and poloidal flow measurement are utilized for the FICXS measurement. Two curved mirrors are installed in one toroidal section of LHD and light is reflected at these mirrors to 48 optical fibers so as to have a better spatial resolution of active CXS measurement with the radial-NB. For the measurement of background, an identical poloidal cross section is viewed but at a different toroidal location, where no interference by both of the tangential- and radial-NBs are expected. In each fiber bundle, half of the fibers are used for the sight lines reflected at the upper mirror and the rest are used for those reflected at the lower mirror. All of the fibers from both bundles are coupled to single spectrometer (BUNKOKEIKI/ HTP-400E) with a readout of a charged coupled device(CCD) camera (ANDOR/ECD-400 DW438-BV). In the data presented in this paper, the integration time of the CCD is set to $150 \mathrm{~ms}$, while the sampling interval is set to $300 \mathrm{~ms}$. The charge exchange components of the spectra are obtained by the subtraction of background spectra measured at the other toroidal location. As can be expected from the geometry shown in Fig. 1(b), the beam emission spectra (BESs) of $H_{\alpha}$ light from the NB is also observed in the spectra. Fortunately all of these BES components are redshifted so we can eliminate these complications by limiting our spectral range to the blue shifted side.

The radial-NB injection causes an additional difficulty in the FICXS measurement because of the energy dependence of charge exchange reaction rate and the large variation of relative velocities between the injected NBs and gyrating fast ions. In Fig. 2(a), the energy dependence of
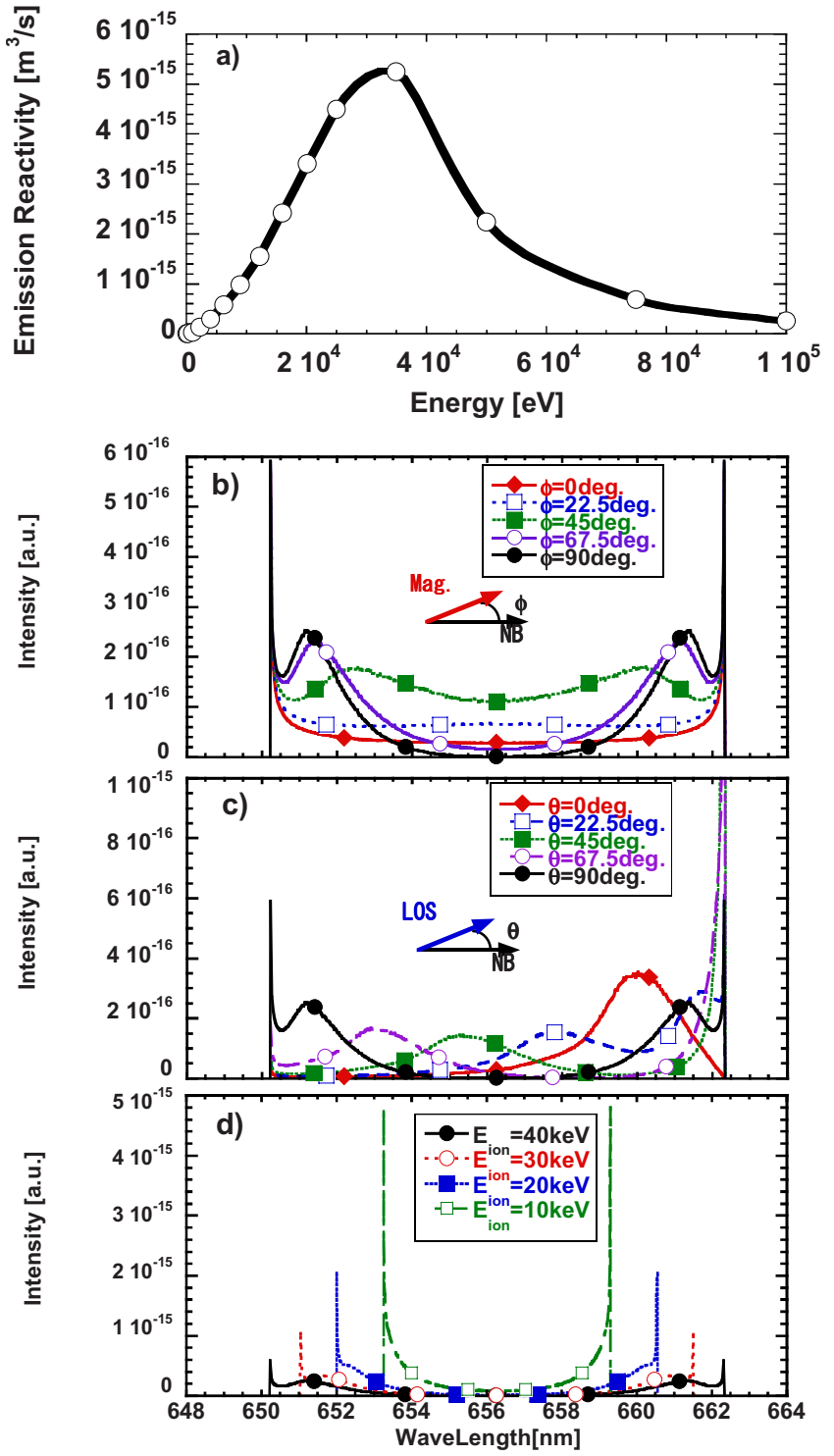

FIG. 2. (Color online) (a) Energy dependence of the charge exchange reaction rate for the hydrogen to hydrogen $(n=3)$ charge transfer process. Variations of the Doppler shifted $H_{\alpha}$-spectra for monoenergetic fast ions with the change in the (b) NB-injection pitch angle, (c)the angle between NB-injection path, and the LOSs, and (d) the energy of gyrating fast ions. The directions of the LOS, the magnetic field lines and the NB-injection can be considered orthogonal to one other if the angle between them is not indicated.

reaction rate is shown for the charge exchange reaction of $\mathrm{H}^{0}+\mathrm{H}^{+}=>\mathrm{H}^{+}+\mathrm{H}^{0}(n=3) .^{5}$ The variation of chargeexchange-driven $H_{\alpha}$ spectra with the change in the NBinjection pitch angle is shown in Fig. 2(b). In calculating the spectra, the energy of gyrating fast ions and NBs was fixed at $40 \mathrm{keV}$. The pitch angles of fast ions are set to $90^{\circ}$. The directions of the lines of sight (LOS) and the magnetic field lines are assumed to be orthogonal to one other. The angle between the LOS and NB-injection path is also assumed to be a right angle in this calculation. As shown in Fig. 2(b), only two large peaks at the edges of the spectra are expected to be observed when the NB is tangentially injected to the magnetic field lines. The shape of the spectra is similar to $H_{\alpha}$ emission spectra from monoenergetic fast neutrals arising from the charge exchange process between the cold neutrals 
and monoenergetic fast ions. However, when the pitch angle of the NB becomes larger than $45^{\circ}$, the situation changes: two other peaks appear between the peaks at the edges. These two additional peaks are produced when the relative velocities of the fast ions to the injected neutrals become $33 \mathrm{keV}$, where the peak of the reaction rate in Fig. 2(a) is located.

As can be seen from the observation geometry in Fig. 1(b), the angle $(\theta)$ between the LOS and NB-injection direction decreases as the observation region goes toward the inboard side of LHD. This variation of $\theta$ also causes a problem when the NB is injected almost perpendicular to the magnetic field lines [see Fig. 2(c)]. The spectra shown in Fig. 2(c) are calculated using similar parameters to the calculations shown in Fig. 2(b), but the pitch angle of the NB is fixed at $90^{\circ}$ and the $\theta$ is scanned. In Fig. 2(c), the spectrum is symmetric at around the wavelength of $656.3 \mathrm{~nm}$ when the $\theta$ is equal to $90^{\circ}$. As $\theta$ decreases, the peaks between the two edges are shifted in the red direction, and the emission in the blue region, especially the line at the edge, decreases.

These effects caused by the radial-NB injection, in addition to the effect of energy variation of fast ions that is shown in Fig. 2(d), make it extremely difficult to obtain the fast ion distribution function from the deconvolution of Doppler shifted $H_{\alpha}$ spectra. Instead, we have decided to use the result of FICXS measurement as a benchmark data of the numerical simulations, such as GNET code. ${ }^{6,7}$

\section{INITIAL EXPERIMENTAL RESULTS AND ITS ANALYSIS}

Figure 3 shows the observed spectra by the FICXS measurement for the discharges of $82642\left(E_{\text {radial-NB }}\right.$ $=37.5 \mathrm{keV}), \quad 82644\left(E_{\text {radial }-\mathrm{NB}}=33.5 \mathrm{keV}\right), \quad$ and $82645\left(E_{\text {radial }-\mathrm{NB}}=29.5 \mathrm{keV}\right)$ at $t=1.3 \mathrm{~s}$. The integration times of the measurements were set to $150 \mathrm{~ms}$ for these discharges. In these discharges, the ion temperatures $\left[T_{i}(0)=\right.$ $\sim 2.2 \mathrm{keV}]$, the electron temperatures $\left[T_{e}(0)=\sim 2.8 \mathrm{keV}\right]$, and line averaged electron densities $\left(n_{e}=\sim 1 \times 10^{19} \mathrm{~m}^{-3}\right)$ were kept at same levels, and only the injected energies of the radial-NBs were scanned. Two types of typical (background subtracted) spectra are shown in Figs. 3(a) and 3(c). The spectra in Fig. 3(a) are obtained from the LOS which crosses close to the diverter $X$-point, while the spectra in Fig. 3(c) are obtained from the LOSs which are viewing the core regions $(r / a=\sim 0.6)$ of the plasma. The major radius of the LOS at the midplane $\left(R_{\text {mid }}\right)$ is $4.42 \mathrm{~m}$ for the former spectra and $4.05 \mathrm{~m}$ for the latter. Since both of the spectra are changing with the variation of the NB-injection energies [especially note that the position of the peak in Fig. 3(a) coincide with the wavelength of Doppler shifted $H_{\alpha}$ light from the neutrals of the injection beam energies] it is confirmed that the observed spectra are due to the CXS spectra of fast ions being produced by the radial-NB.

Since the spectra in Fig. 3(a) do not show any dips due to the energy dependence of the emission reaction rate, we can determine that the fast neutrals were created by the charge exchange reactions between the cold neutrals and fast ions. Usually the emission due to this charge exchange pro-
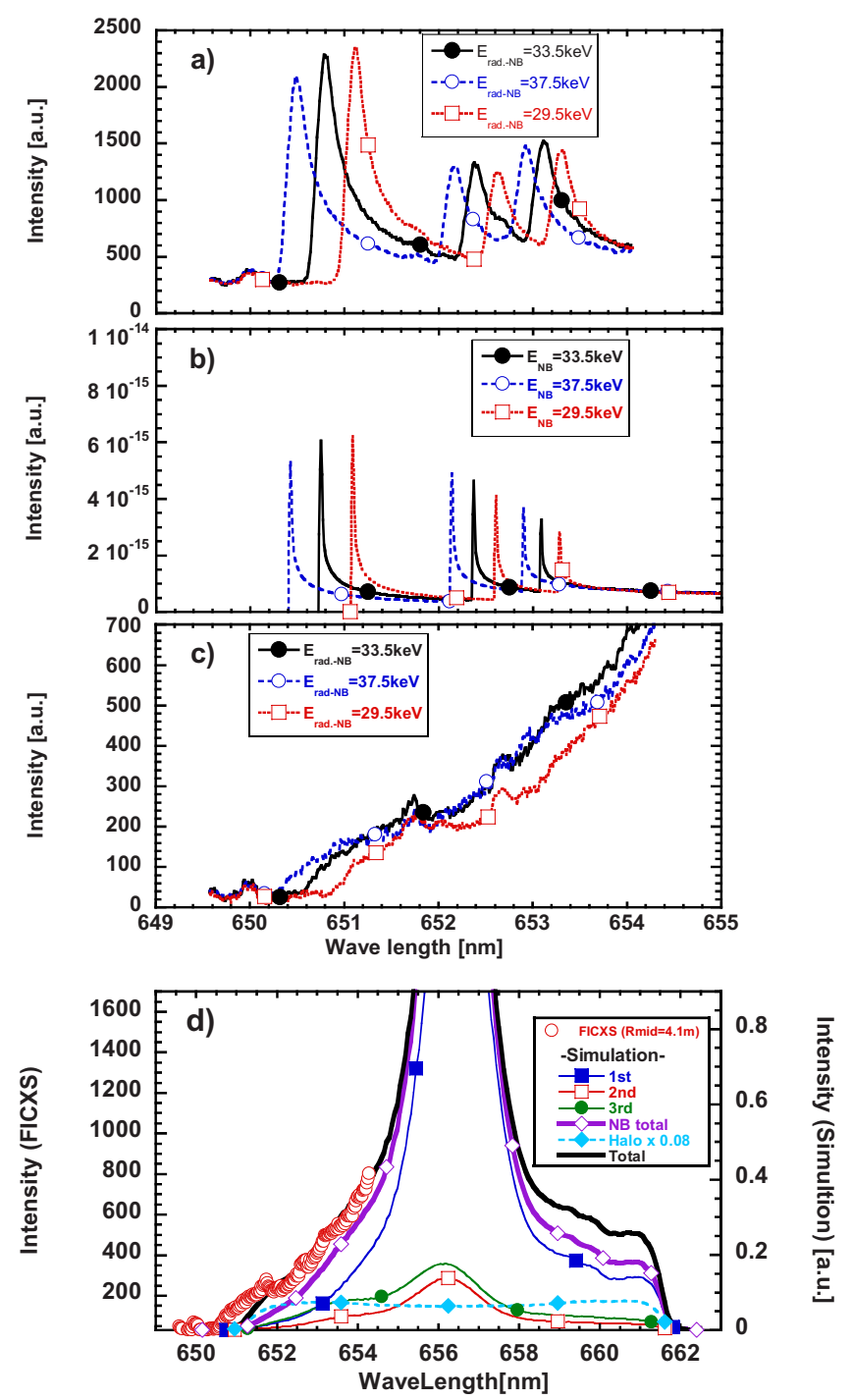

FIG. 3. (Color online) Variations of FICXS spectra with the change in the radial-NB injection energies. (a)Typical experimentally obtained spectra for the LOS observing close to the diverter $X$-point $\left(R_{\text {mid. }}=4.4 \mathrm{~m}\right)$, (b) calculated FICXS spectra for NB-injected fast ions without slowingdown process, and (c) experimental FICXS spectra for core regions $\left(R_{\text {mid }}\right.$ $\left.=4.1 \mathrm{~m}, r / a_{\text {mid }}=0.642\right)$ are shown. Peaks at around 650, 651.7, and $652.7 \mathrm{~nm}$ in (a) and (c) are considered to be due to the spectral lines of impurity ions. (d) Comparison of the GNET simulated spectra to the FICXS $\operatorname{spectra}\left(E_{\text {radial-NB }}=33.5 \mathrm{keV} / \mathrm{shot}\right.$ No. $\left.82644, t=1.3 \mathrm{~s}\right)$.

cess is background subtracted. However, this is not the case for this spectra since the LOS in Fig. 3(a) observes mostly outside of the last closed flux surface at the NB-injected toroidal section and most of the fast ions which cross this LOS are promptly lost; these particles are highly localized in the toroidal section where they were ionized and lost. Therefore, they cannot be detected at the toroidal section where the background channels are installed. Calculated FICXS spectra for fast ions of three energy components $\left(E_{\mathrm{nb}}, E_{\mathrm{nb}} / 2\right.$, and $\left.E_{\mathrm{nb}} / 3\right)$ are shown in Fig. 3(b). The fraction of each energy component is assumed to be same, i.e., $1 / 3$, in this figure. The peak positions of FICXS spectra in the figure agree quite well with those in Fig. 3(a).

A comparison of one of the spectra (82644) in Fig. 3(c) with the results of GNET calculations is shown in Fig. 3(d). GNET (Ref. 7) is a Monte Carlo simulation code which solves 
a drift kinetic equation in five-dimensional (5D) phase space (three dimensional in real space and two dimensional in velocity space). In the calculation shown here, the velocity distribution functions of fast ions produced by the radial-NB are also calculated in the 5D phase space, but their spatial information are averaged over the same minor radius locations (flux averaged). In evaluating the $H_{\alpha}$ emission spectra from the GNET calculations, the information of attenuated NB fractions $\left(f_{\mathrm{NB}, j} \gamma_{\mathrm{NB}, j}\right)$ for each beam component and the fraction $\left[f_{\text {halo }}(\vec{r})\right]$ of halo neutrals, which are produced by the charge exchange reaction between bulk ions and injected neutrals, are necessary. Here, the $f_{\mathrm{NB}, j}$ corresponds to the fraction of $j$ th component $(j=1-3)$ of the NB at the injection port and the $\gamma_{\mathrm{NB}, j}$ is the attenuation factor of each NB component in the LHD plasma. The beam fractions are roughly $\left(f_{\mathrm{NB}, 1}, f_{\mathrm{NB}, 2}, f_{\mathrm{NB}, 3}\right)=(0.7,0.1,0.2)$ for the radial-NB injector. The attenuation factors $\left(\gamma_{\mathrm{NB}, j}\right)$ are calculated using a NB attenuation code, which are developed at NIFS, using the ADAS system. ${ }^{8}$ The distributions of halo neutral fractions are evaluated using

$$
f_{\text {halo }}(\vec{r})=n_{\text {halo }}(\vec{r}) / n_{\mathrm{NB}}(\vec{r})=\sum_{j=1}^{3} \frac{\left\langle\sigma_{\mathrm{cx}} \nu\right\rangle_{i j} f_{\mathrm{NB}, j} \gamma_{\mathrm{NB}, j}}{\left\langle\sigma_{\mathrm{ei}} \nu\right\rangle_{\mathrm{ei}}+\left\langle\sigma_{\mathrm{ii}} \nu\right\rangle_{\mathrm{ii}}},
$$

where the $\sigma_{\mathrm{cx}}, \sigma_{\mathrm{ei}}$, and $\sigma_{\mathrm{ii}}$ are cross section for hydrogen neutrals of charge exchange reaction with hydrogen ions, that of electron impact ionization, and ion impact ionization, respectively. The \langle\rangle$_{\mathrm{ei}}$ and \langle\rangle$_{\mathrm{ii}}$ indicate the integration over the Maxwell distribution functions. The \langle\rangle$_{\mathrm{ij}}$ indicates integration over the Maxwellian and beam distribution function. Since the beam energy is much larger than the ion temperature in LHD plasmas, we have used a cold plasma approximation. The $\sigma_{\mathrm{cx}}$ and $\sigma_{\mathrm{ii}}$ are obtained from Ref. 9, and the $\left\langle\sigma_{\mathrm{ii}} v\right\rangle_{\mathrm{ii}}$ is calculated by the numerical integration of $\sigma_{\mathrm{ii}} \nu$ with Maxwell distribution function, while the $\left\langle\sigma_{\mathrm{ei}} v\right\rangle_{\mathrm{ei}}$ is obtained from the AMDIS database. ${ }^{5}$ Equation (1) includes the balance equation of halo neutrals but does not include the transport effect of these neutrals therefore the fraction evaluated by the equation overestimates the fraction of halo neutrals. Hence, an adjusting constant of $\varepsilon_{\text {halo }}$ is introduced to find the reasonable value of the halo neutral fraction, i.e., the halo neutral fraction distribution along the LOS of the measurement was evaluated according to the Eq. (1), but its line-integrated values are adjusted by $\varepsilon_{\text {halo }}$.

The FICXS spectra obtained by the simulation shows the asymmetry at around the wavelength of $656.28 \mathrm{~nm}$, as shown in Fig. 3(c). This asymmetry is due to the variation of the $H_{\alpha}$ spectra with the change of $\theta$, which was discussed in the Sec. II.
It turns out that the spectra from the experiment cannot be reconstructed from the neutral contribution of the radialNB alone; the contribution of halo neutrals must be taken into account (especially for the hump below $652.5 \mathrm{~nm}$ ). Using an adjustment parameter of $\varepsilon_{\text {halo }}=0.08$ gives good agreement between the experiment and the simulation. In the spectra obtained by the experiment, another small hump in the range between 652.5 and $653.8 \mathrm{~nm}$ is also observed. Comparing the shape of this hump with each contribution of neutral component in the simulations, the FICXS spectra with the third-energy component of the radial-NB seems to have a role in this spectral range. More detailed evaluation of NB fraction at the injection port seems to be necessary taking into account the operational condition of the ion sources of the radial-NB injector. To have further investigations, better evaluation of halo neutral transport using Monte Carlo code, such as DEGAS, ${ }^{10}$ is necessary.

\section{ACKNOWLEDGMENTS}

The authors wish to thank the support of the LHDexperimental group in performing the experiments shown in this paper. We also wish to thank the continuous encouragement by the Director general of NIFS, Professor Motojima. This work was supported by NIFS2006/KLBB301, NIFS2007/ULBB503, NIFS2007/ULBB510, and NIFS2007/ ULBB511, and partly supported by the Grant-in-Aid for Scientific Research (A), No. 18206094 and (B), No. 18340189.

\footnotetext{
${ }^{1}$ A. Sagara, S. Imagawa, O. Mitarai, T. Dolan, T. Tanaka, Y. Kubota, K. Yamazaki, K. Y. Watanabe, N. Mizuguchi, T. Muroga et al., Nucl. Fusion 45, 258 (2005).

${ }^{2}$ M. Osakabe, T. Yamamoto, Y. Takeiri, T. Mutoh, E. Asano, K. Ikeda, K. Tsumori, O. Kaneko, K. Kawahata, N. Ohyabu et al., Rev. Sci. Instrum. 72, 788 (2001).

${ }^{3}$ M. Osakabe, S. Murakami, T. Seki, Y. Takeiri, M. Sasao, K. Tanaka, K. Narihara, and LHD Experimental Group, Rev. Sci. Instrum. 75, 3601 (2004).

${ }^{4}$ W. W. Heidbrink, K. H. Burrel, Y. Luo, N. A. Pablant, and E. Ruskov, Plasma Phys. Controlled Fusion 46, 1855 (2004).

${ }^{5}$ CHART and AMDIS databases from NIFS atomic and molecular databases (http://dbshino.nifs.ac.jp/).

${ }^{6}$ S. Murakami, U. Gasparino, H. Idei, S. Kubo, H. Maassberg, N. Marushchenko, N. Nakajima, M. Romé, and M. Okamoto, Nucl. Fusion 40, 693 (2000).

${ }^{7}$ S. Murakami, H. Yamada, M. Sasao, M. Isobe, T. Ozaki, T. Saida, P. Goncharov, J. F. Lyon, M. Osakabe, T. Seki et al., Fusion Sci. Technol. 46, 241 (2004).

${ }^{8}$ H. P. Summers, The ADAS User Manual, version 2.6, 2004 (http:// adas.phys.strath.ac.uk/).

${ }^{9}$ R. Ito, T. Tabata, S. Shirai, and R. A. Phaneuf, Report Nos. JAERI-M 93-117 and JAERI-DATA 95-008.

${ }^{10}$ D. B. Heifetz, Physics of Plasma-Wall Interaction in Controlled Fusion, edited by D. E. Post and R. Behrisch (Plenum Press, New York, 1986).
} 